\title{
Residual Deformation Control Design for Car Handbrake Operating Arm Assembly
}

\author{
Yuan $\mathrm{Li}^{1}$ and $\mathrm{Li} \mathrm{Ma}^{2}$ \\ ${ }^{1}$ School of Automotive Engineering, Wuhan Huaxia University of Technology, Wuhan, China \\ ${ }^{2}$ School of Automotive Engineering, Wuhan University of Technology, Wuhan, China
}

\begin{abstract}
The aim of the paper is to control residual deformation of the handbrake arm assembly under lateral force in an effective way. Compared with the test method, nonlinear FEA method is higher in accuracy and fewer in cost of test specimen, which is an available means to control the residual deformation before producing.
\end{abstract}

\section{Introduction}

Handbrake is an important safety car assembly. Under lateral and longitudinal forces, handbrake will be in large displacement or unrecoverable residual deformation, or even occurs partial fracture, which seriously affect its performance. Foreign references specifiy acceptable residual deformation. The handbrake companies often use test method to get deformation datas, but it needs huge amount of samples, which make waste of materials and increase of costs. The paper provide another mothed of controlling residual deformation to decrease the cost and waste of resource.

\section{Tensile test of handbrake operating arm}

There is a quality standard to check the strength and stiffness of the handbrake arm assembly, which mentions that the lateral offset of the arm end should not exceed $30 \mathrm{~mm}$ if the maximal load on the arm reaches $400 \mathrm{~N}$, and the residual offset should be less than $10 \mathrm{~mm}$ when unloading. A lateral tensile test is designed to test whether the strength and stiffness is up to standard.

As the lateral clearance tester shown in Figure 1, the sample of the handbrake arm, which was fixed on the positioning fixture of tester, located at the same position with the actual condition, and the arm rotated to a certain angle according to the specification. The force sensor connected assistor fixed on the test bench with one end, another end of which jointed contacting device by nuts, and its signal were output on the digital screen. Electronic display vernier cliper were also fixed on the test bench, and parallel to the tester's surface. The load was applied on the screw assistor until the contacting device touched the arm end. When the depth ruler of the vernier caliper was moved to the position that just touched the contacting chip--the minimal data were shown on the caliper, reset the caliper and unload the lateral force.

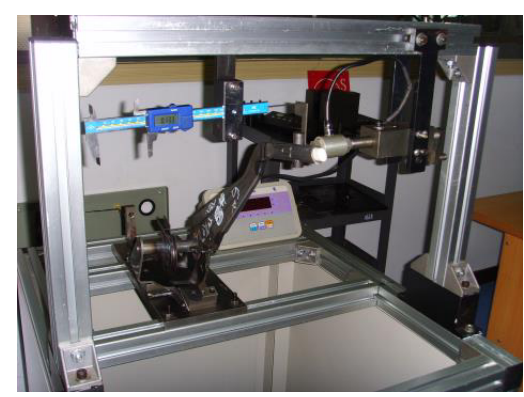

Fig.1. Lateral clearance tester

The lateral force test started after pre-adjustment when illustrated above. First,when contacting chip contacted with the arm after screw rotating and the lateral force was $5 \mathrm{~N}$, the displacement on the caliper was recorded and all parts of the assembly were not loose. Unloading the lateral force and rotating the screw to make two parts contact again,the load was applied until the lateral force peaked $100 \mathrm{~N}$, and the caliper data was recorded. Applying load as the same steps as above until the lateral force reaches $400 \mathrm{~N}$, record the lateral displacement $L_{p}$ (Y residual offset on the arm end) and unload the 
force.we also find that there was no loose phenomenon between different parts. Finally, changed the location of the loading device and the measuring equipment,and test again to get the negative side lateral force results.

\section{FEA control design for the residual deformation of handbrake operating arm}

Although the offset and residual offset in the lateral tensile conditions can be measured by test, there are several problems in the testing process and results. After the positive lateral force test, the residual deformation of the handbrake occurs. Using this assembly again to carry out the negative force test would result in error, because the state of the assembly with plastic deformation is different from which in the specification. To reduce the error in negative force test, it is necessary to use a new assembly that may inceasing testing cost and material consumption. To avoid this error, another method like FEA method can be used.

\subsection{The geometry model and finite element meshing}

Compared with the beam or shell model, 3D solid model is more available to detect the slight displacement and contacting deformation between parts. Therefore, it is better to use 3D solid model of the handbrake arm assembly for FEA(Finite Element Analysis).

Four-node solid element is a reasonable type to ensure high accuracy for the FEA model in finite computing time and scale. Because this FEA model is a nonlinear problem,including nonlinear of state and material, as contacting state changes in lateral tensile process and the material characteristic curve is not linear.Consequently, there are 4034000 solid elements and 10000 nodes meshed in FEA model. Partial enlarged figure of meshing model is shown in Figure 3.

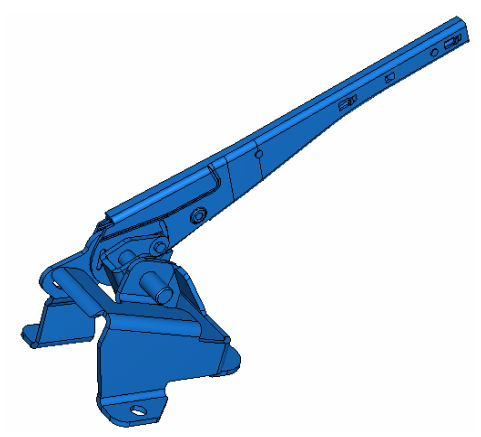

Fig.2. 3D model of the assembly

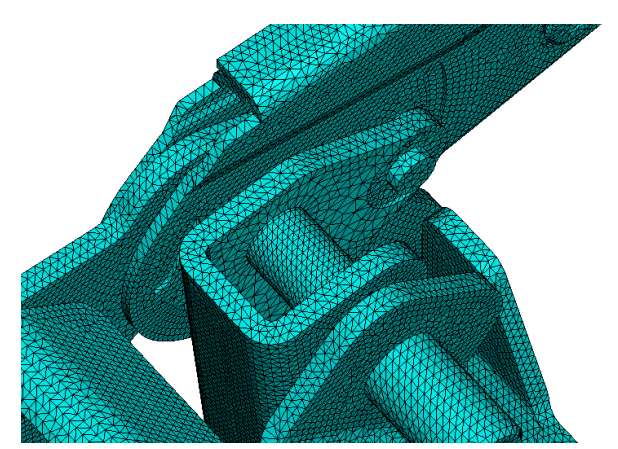

Fig.3. Partial enlarged figure of meshing model

\subsection{Material model}

Considering the specification focus on the residual deformation causing by the lateral tensile force, material nonlinear problem ought to be taken into consideration. The reason is that the residual plastic deformation would occur and the stress-plastic curve of material would be no more linear provided that the stress exceeded the material yield limit. In 
principle, nominal stress-nominal strain curves obtained by the material axial tensile test should transform to true stress-plastic strain curves. The relationship between nominal stress and true stress is

$$
\sigma=\sigma_{\text {nom }}\left(1+\varepsilon_{\text {nom }}\right)
$$

And the relationship between nominal strain and plastic strain is

$$
\varepsilon_{\text {mon }}=\varepsilon_{t}-\sigma / E
$$

In these formulas, $\sigma$-true stress, $\sigma_{m o n}$-nominal stress, $\varepsilon_{m o n}$-nominal strain, $\varepsilon_{p l}$-plastic strain, $\varepsilon_{t}$-true strain, E-elastic modulus

According to the formula (1) and (2), the true stress-plastic strain curve of main material of handbrake arm assembly is shown in Figure 4.

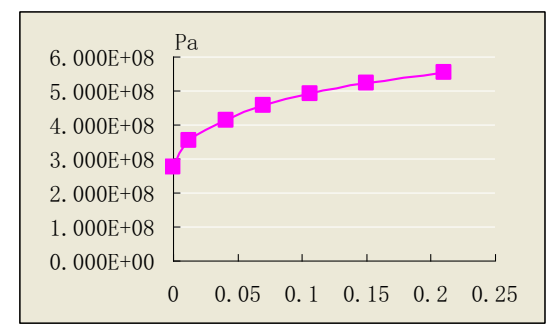

Fig.4. True stress-plastic strain curve of main material of handbrake arm assembly

\subsection{Setting up the contact pairs}

There are two main kinds of contacting pairs between parts of handbrake arm assembly. One is constant contact, which are riveting, welding and articulated relations. Another is variational contact . In FEA, contact is a complex state nonlinear issue. So it is necessary to set different relationships in relative kinds of contact to ensure the nonlinear calculation convergence within a controllable time.

The connection between the rivet and the manipulation arm, as well as little rivet and the cover is riveting; the connection between tooth base and the stationary seat, as well as between the pin shaft and the cover is welding;the connection between pin shaft and it mating parts is articulation. All these relationships can be set to constraint.

While another relationship varies with the increase of the tensile force, such as contact between the convex surface on the cover and the square limited convex surface on the manipulation arm. So sliding contacts are applied on these surfaces.

\subsection{Definition of boundary condition}

To simulate the screw fastening of the handbrake arm assembly, set the nodes near the areas of 3 bolt holes on the tooth plate and the base constraint.

According to the specification, the lateral load of $400 \mathrm{~N}$ is applied on the end of the manipulator arm, which is decomposed into 4 points on the top of arm and cover to avoid local stress concentration caused by load on one single point.

\section{Results contrast between FEA and test}

Table 1. Results in FEA and test method $\quad(\mathrm{mm})$

\begin{tabular}{|c|c|c|}
\hline \multirow{2}{*}{ Test name } & \multicolumn{2}{|c|}{ FEA results } \\
\cline { 2 - 3 } & Deformation & $\begin{array}{c}\text { Residual } \\
\text { deformation }\end{array}$ \\
\hline Positive lateral force & 25.78 & 7.122 \\
\hline Negative lateral force & 29.04 & 9.346 \\
\hline Test name & \multicolumn{2}{|c|}{ Test result } \\
\hline
\end{tabular}




\begin{tabular}{|c|c|c|}
\hline & $\begin{array}{c}\text { Loading } \\
\text { offset }\end{array}$ & Loaded offset \\
\hline Positive lateral force & 38.4 & 6.62 \\
\hline Negative lateral force & 39.32 & 7.04 \\
\hline
\end{tabular}

The results(Table 1) of offset and residual offset in positive/negative lateral force test are achieved by FEA and testing method. From Table 1, the offsets when loading in two conditions are $38.4 \mathrm{~mm}$ and $39.32 \mathrm{~mm}$, both of which outnumber $30 \mathrm{~mm}$ the specification required,showing that the loading offset does not meet the standard.And the residual offsets, which are $6.62 \mathrm{~mm}$ and $7.04 \mathrm{~mm}$ respectively, are under $10 \mathrm{~mm}$.

Comparing the results between the calculation and the tests in lateral force conditions, the displacement and residual deformation by the nonlinear FEA method are much close to the test results, which show that this residual deformation controlling method is effective.

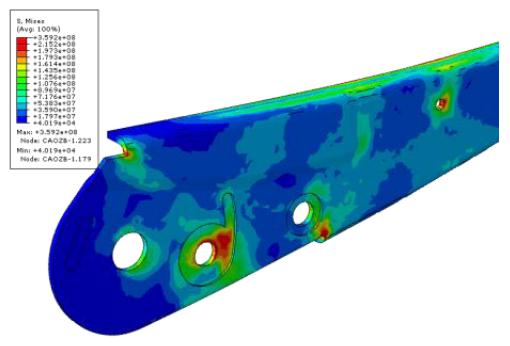

Fig.5. The arm residual stress nephogram

In addition, the lateral force test can measure the displacement and residual deformation on the end of the arm, but

whether the assembly occurs fracture can be only achieved by macroscopic observation, while the internal surfaces of contacting pairs are hard to recognise. Nevertheless, not only the residual stress but also the failure position can be easily obtain via nonlinear FEA method. The residual stress nephogram ot the arm is shown in Figure 5, which demostrates that the residual stress in the shape corner in the upper area are $218.7 \mathrm{MPa}$ and the lower $359.2 \mathrm{MPa}$,both of which are higher than the yield limit. Therefore replacing the corner area from sharp to smooth is a suggested way to reduce the partial residual stress.

\section{Application of the new product design by nonlinear FEA method}

Table 2. The results of W2 assembly in FEA and test method ( $\mathrm{mm}$ )

\begin{tabular}{|c|c|c|}
\hline \multirow{2}{*}{ Test name } & \multicolumn{2}{|c|}{ FEA results } \\
\cline { 2 - 3 } & Deformation & $\begin{array}{c}\text { Residual } \\
\text { deformation }\end{array}$ \\
\hline Positive lateral force & 21.88 & 2.367 \\
\hline Negative lateral force & 15.66 & 1.906 \\
\hline \multirow{2}{*}{ Test name } & \multicolumn{2}{|c|}{ Test result } \\
\cline { 2 - 3 } & $\begin{array}{c}\text { Loading } \\
\text { offset }\end{array}$ & Loaded offset \\
\hline Positive lateral force & 20.48 & 3.07 \\
\hline Negative lateral force & 15.57 & 4.85 \\
\hline
\end{tabular}


This study of nonlinear FEA method applied to a new product W2 handbrake arm assembly. The test and FEA results of W2 are shown in Table2, showing the approaching of the results between these two methods and high precision in the FEA nonlinear method to control the residual deformation.

\section{Conclusion}

The nonlinear FEA method to control the residual deformation of the handbrake operation arm under the lateral force has achieved good results. If the residual deformation is controlled before test, it can effectively reduce low accuracy results in test method and waste of resources by trial and error. Modifing the product's design according to the nonlinear FEA results, the structure will be improved effectively with less time in designing, producing and testing.

\section{References}

1. J.Xiao.Transpo World,6,42(1998)

2. R.A.C.Slater.Engineering plasticity. (Thomson Press, 1977)

3. Delphi Corporation. Automotive Engineering,( 2005)

4. Si.Xue.Elastic-plastic mechanics. (China buliding material press, 2005)

5. Y.Guo, W.Tao, Z.Zhuang. Linear and nonlinear finite element. (Mechanical Industry Press: Beijing, 2005) 